\title{
Schon Kinder leiden unter Schlafapnoe
}

\section{Ca. $4 \%$ aller Kinder leiden am Ob- struktiven-Schlafapnoe-Syndrom (OSAS). In manchen Fällen tritt das OSAS im Zusammenhang mit einer schweren Adipositas oder im Rah- men eines Down-Syndroms auf. In diesen Fällen kommt häufig die CPAP-Therapie zum Einsatz.}

- Die Nicht-Behandlung des OSAS kann bei Kindern verschiedene Folgen haben, die sich z. T. von denen der erwachsenen OSAS-Patienten unterscheiden: Meistens fallen die Kinder nämlich nicht primär durch Müdigkeit auf, sondern vielmehr durch Verhaltensstörungen, wie dem sog. Aufmerksamkeitsund Hyperaktivitätsdefizit (ADHD)Syndrom.

In eine prospektive Studie wurden 52 Kinder und Jugendliche eingeschlossen. Die Kinder litten u.a. unter Adipositas (69\%), genetischen Syndromen (17\%), ZNS-Erkrankungen (11\%), kranio-fazialen Dysmorphien (5\%), Lungenerkrankungen (5\%), Wachstumshor-

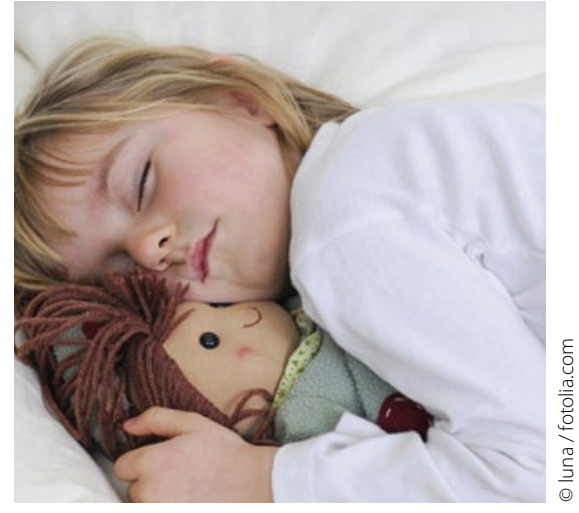

Ca. $4 \%$ aller Kinder haben eine obstruktive Schlafapnoe.

mon-Defiziten (2\%) und Entwicklungsstörungen (19\%).

Alle Patienten erhielten eine Ausgangs-Polysomnografie. Danach erfolgte eine 2-Wochen-Periode der Gewöhnung an die CPAP-Therapie, gefolgt von einer CPAP-Titration sowie einer Kontroll-Polysomnografie unter CPAP nach drei Monaten. Zum Zeitpunkt der Ausgangs-Messung und am Ende der

dreimonatigen CPAP-Phase wurden verschiedene Fragebögen zur Schläfrigkeit, zum Verhalten, und zur Lebensqualität ausgefüllt. Die CPAP-Therapie führte bei den Kindern und Jugendlichen mit OSAS zu einer signifikanten Verbesserung von Verhaltensstörungen.

\section{Kommentar}

Die Studie belegt, dass Verhaltensstörungen, Tagesschläfrigkeit und Einschränkungen der Lebensqualität bei kindlichen und jugendlichen OSAS-Patienten sehr positiv durch die CPAP-Therapie beeinflusst werden können. "Verhaltensauffällige" oder an vermehrter Tagesschläfrigkeit leidende Schulkinder sollten unbedingt einer schlafmedizinischen Diagnostik und Therapie zugeführt werden sollten.

K. RASCHE =

- C. L. Marcus et al.

Effects of Positive Airway Pressure Therapy on Neurobehavioral Outcomes in Children with Obstructive Sleep Apnea. Am. J. Respir. Crit. Care Med. 185 (2012) 998-1003

\section{Hilft Vitamin C gegen Erschöpfung?}

\section{Ein nebenwirkungsarmes, legales Mittel gegen Erschöpfung glauben koreanische Forscher gefunden zu haben.}

- Die Forscher randomisierten 141 gesunde Büroangestellte in zwei Gruppen. Die Experimentalgruppe erhielt eine einmalige Dosis von 10g Vitamin C intravenös verabreicht, während die Kontrollgruppe $\mathrm{NaCl}$ i.v. erhielt.

Zwei und 24 Stunden nach dieser Therapie wurde die Erschöpfung beziehungsweise Müdigkeit der Probanden quantifiziert. Die Auswertungen zeigen, dass im Vergleich zur Kontrollgruppe die mit Vitamin C behandelten Probanden signifikant weniger Erschöpfung/ Müdigkeit angaben. Ernste Nebenwir- kungen waren nicht zu beklagen. Eine Subgruppenanalyse deutet ferner darauf hin, dass der Effekt bei solchen Personen besonders deutlich ausgeprägt war, die initial einen niedrigen VitaminC-Spiegel hatten. Die Autoren ziehen aus diesen Daten den Schluss, dass Vitamin $C$ i.v. den Erschöpfungsgrad gesunder Personen reduziert

\section{Kommentar}

Wäre es nicht wunderbar, wenn wir ein Mittel gefunden hätten, das ohne Nebenwirkungen unsere Leistung steigern kann? $\mathrm{Ob}$ Vitamin C dieses Wundermittel ist, möchte ich allerdings bezweifeln. Auf den ersten Blick ist die vorliegende Studie gut gemacht; bei genauerem Hinschauen kommen mir allerdings Zweifel an ihrer Validität. Z. B. ist zu beanstanden, dass der Hauptzielparameter, die Erschöpfung/Müdigkeit, nicht mit einer validierten Methode erfasst wurde. Aus meiner Sicht wiegt vielleicht noch schwerer, dass in der Kontrollgruppe so gut wie kein Placeboeffekt beobachtet wurde. Ich hätte erwartet, dass in dieser Prüfsituation ein recht deutlicher Effekt alleine aufgrund der Erwartungshaltung der Probanden zu erwarten gewesen wäre.

Die intravenöse Gabe von Vitamin C gehört mit zu den umstrittensten Therapieformen überhaupt. Die vorliegende Studie trägt nur wenig dazu bei, die Vitamin-C-Kontroverse zu beenden.

E. ERNST .

- S. Y. Suh et al.

Intravenous vitamin $C$ administration reduces fatigue in office workers: a double-blind randomized controlled trial. Nutr. J. 11 (2012) 7 